\title{
I GIURISTI UMANISTI FRANCESI E IL CORPUS IURIS ANTEIUSTINIANI ${ }^{1}$
}

\author{
Nota del m.s. JEAN-LOUIS FERRARY (*)
}

(Adunanza del 6 ottobre 2011)

SunTO. - La riscoperta di testi giuridici anteriori al Corpus iuris civilis giustinianeo è un aspetto importante dell'attività dei giuristi umanisti, sopratutto francesi perche i manoscritti utilizzati si trovavano sopratutto in biblioteche capitolari e monastiche del regno di Francia. Ma il ius anteiustinianum non era un corpus omogeneo, e la sua edizione poneva problemi molto complessi, che hanno ricevuto soluzioni diverse dal XVI al XIX secolo.

$* * *$

ABSTRACT. - Rediscovery of legal texts earlier than Justinian's Corpus Iuris Ciuilis is an important part of the activity of humanist jurists, mainly of French ones, because most of related manuscripts were preserved in capitular and monastic libraries within the kingdom of France. But ius anteiustinianum was in no way a homogeneous corpus, so that its edition raised very complex problems, which were answered in different ways from the $16^{\text {th }}$ to the $19^{\text {th }}$ century.

(*) École Pratique des Hautes Études (Parigi) - AnHiMA UMR 8210.

1 Vorrei esprimere la mia profonda gratitudine per l'Istituto Lombardo che $\mathrm{mi}$ ha eletto tra i sui membri stranieri. L'onore dell'ingresso nell'illustre compagnia mi è tanto più gradevole che è, se posso dire, l'apice di una già lunga e feconda collaborazione colle università di Milano, e più regolarmente ancora coll'università e l'Istituto Universitario di Studi Superiori di Pavia. 
Il diritto giustinianeo (ius Iustinianum) era un insieme coerente e ben identificabile: formato dalle Istituzioni, dal Digesto e dal Codice, privava di vigore le compilazioni anteriori e tutte le fonti giuridiche che avevano circolato fino ad allora. Fu emanato da Giustiniano fra il 529 e il 534, per essere poi completato dalle Novelle, vale a dire le costituzioni posteriori alla promulgazione del Codice. Fu proprio questo insieme di testi, seppur un poco mutilato e alterato dalla tradizione manoscritta, arricchito da un piccolo numero di testi medievali e soprattutto provvisto dell'apparato di commento della Glossa, che costituì il nocciolo del ius civile, cioè del diritto applicato in gran parte dell'Europa a partire dal dodicesimo secolo. Per definire questo insieme di testi raccolti da Giustiniano si parlò appunto di Corpus iuris civilis.

L'umanesimo giuridico, pur senza mai respingere completamente i contributi dati dai glossatori e dai commentatori all'interpretazione del Corpus iuris civilis, si distinse in primo luogo per la volontà di un ritorno più fedele ai testi, a cominciare proprio dai testi del Corpus iuris civilis. Perciò, per stabilire il testo del Digesto, gli umanisti ricorsero al manoscritto più importante e fedele, fino ad allora poco utilizzato, ossia le famose Pandette Fiorentine; provvidero poi a reintrodurre in tutto il Corpus iuris dei testi scritti in greco, che nel medioevo erano stati sostituiti con traduzioni latine o più semplicemente erano stati omessi, in particolare nel Codice, la cui ricostituzione fu compiuta non prima del 1567 . $^{2}$

A fronte del Corpus iuris civilis giustinianeo venne formandosi, dal Cinquecento all'Ottocento, ${ }^{3}$ quasi specularmente, un Ius anteiustinianum, cioè per così dire il gruppo dei testi del diritto romano anteriori alla compilazione giustinianea, che erano stati sì abrogati da Giustiniano, ma che in parte erano sopravvissuti.

L'edizione del ius anteiustinianum poneva dei problemi filologici in parte analoghi, ma a queste difficoltà s'aggiungeva il fatto che esso, a differenza del Corpus iuris giustinianeo non aveva mai costituito un insieme omogeneo. E' delle vicende che portarono prima a raccogliere questi testi, e poi a disperderli in varie raccolte, vicende che si

2 A. Agustín, Constitutionum graecarum Codicis Iustiniani Imp. collectio et interpretatio, Ilerdae, P. Roburius, 1567.

3 Cfr. J. M. COMA FORT, Ius civile anteiustinianeum. Indice comentado de las colecciones de fuentes del Corpus iuris civilis, Cizur Menor, 2008. 
dipanano nell'arco di tre secoli che intendo parlare oggi. Si tratta di vicende editoriali che hanno un interesse sostanziale, perché mostrano come si sia modificata nel tempo la percezione di questi testi.

Il primo testo che fu riscoperto fu la lex Romana Visigothorum (correntemente chiamato anche Breviario di Alarico): questa raccolta, promulgata nel 506 perché se ne servissero i Romani che vivevano sotto la dominazione dei re visigoti nella penisola iberica e in una parte della Gallia, comprendeva sia costituzioni imperiali sia testi di giuristi romani. Le costituzioni imperiali venivano dal Codice Teodosiano (emanato nel 438) e dalle Novelle posteriori alla promulgazione di esso; gli scritti dei giuristi erano invece rappresentati da un epitome delle Istituzioni di Gaio, da brani delle Sentenze di Paolo, dei Codici Gregoriano e Ermogeniano (che sono raccolte private di costituzioni anteriori al Teodosiano) e da un brano dei responsa di Papiniano. Tranne l'Epitome di Gaio, tutti questi testi erano accompagnati da una Interpretatio che aveva la duplice funzione di chiarirli e di adattarli al diritto romano allora in vigore.

Il testo completo della lex Romana fu edito nel 1528 da Johann Sichard, sotto il titolo ingannevole di Codex Theodosianus: ${ }^{4}$ ingannevole perché è vero che la lex Romana Visigothorum aveva attinto dal Codice Teodosiano, ma appunto molte costituzioni di questo codice erano rimaste escluse dalla raccolta di Alarico. Dei 16 libri del Codice promulgato da Teodosio II nel 438 per tutto l'impero romano, così come delle Novelle successive, Sichard non conosceva altro che gli estratti che Alarico aveva incluso nel suo Breviario. In realtà, qualche manoscritto che conteneva interi libri del Codice Teodosiano era sopravvissuto, e altre costituzioni, del Codice stesso o Novelle, erano state copiate in appendice ad alcuni manoscritti della lex Romana. La quasi totalità di questi manoscritti fu ritrovata proprio nel corso del Cinquecento, essenzialmente nelle biblioteche monastiche o capitolari del regno di Francia.

A partire dal 1550, Jean du Tillet poté perciò pubblicare una

4 J. SICHARD, Codicis Theodosiani libri XVI, quibus sunt ipsorum principum auctoritate adiectae Nouellae Theodosii, Valentiniani, Martiani, Maioriani, Seueri. Caii Institutionum lib. II. Iulii Pauli receptarum sententiarum lib. V. Gregoriani Codicis lib. V, Hermogeniani lib. I, Papiniani tit. I. Hiis nos adiecimus ex uetustissimis bibliothecis, eo quod ad ius ciuile pertinerent, et alterius etiam responsa passim in Pandectis legerentur, L. Volusii Metiani lib. de asse, Iulii Frontini lib. de controuersiis limitum cum Aggeni Vrbici commentariis, Basileae, H. Petrus, 1528. 
edizione che conteneva il testo originale dei libri da 9 a 16 del Codice Teodosiano, pur con delle mutilazioni nel libro 16, e migliorare il testo dei primi otto libri del Codice stesso. ${ }^{5}$ Nel 1566, nella sua prima edizione del Tedosiano, Cujas (Cuiacio) poté pubblicare il testo originale dei libri 6, 7 e 8 e il testo completo del libro 16, senza contare i molti importanti miglioramenti che poté introdurre grazie ad altri manoscritti, ai quali poté avere accesso in particolare grazie alla collaborazione di Pierre Pithou. ${ }^{6}$ Quest'ultimo pubblicò a sua volta nel 157142 Novelle inedite. ${ }^{7}$ Infine una seconda edizione del Teodosiano pubblicata nel 1586 senza il nome dell'editore e senza prefazione, ma con il privilegio del Cuiacio, fece segnare ancora altri progressi: ${ }^{8}$ vedremo più avanti quale ruolo abbia avuto in quest'edizione Pierre Pithou, insieme a suo fratello François.

5 J. DU TILLET, E libris constitutionum Theodosii A. libri priores octo longe meliores quam adbuc circunferebantur, sed ab Alarico rege Gothorum ita deminuti, ut uix decima pars in his hodie appareat eorum, quae in Theodosiano codice continebantur. Posteriores octo integri, nunc primum post $M$ annos in lucem reuocati a Io. Tilio Engolism., Parisiis, apud C. Guillard et G. Desbois, 1550.

6 Codicis Theodosiani libri XVI quam emendatissimi, adiectis quas certis locis fecerat Aniani interpretationibus. Ex his libris nunc primum integri prodeunt VI. VII. VIII. XVI. Ceteri aucti sunt innumeris Constitutionibus. Nouellarum Theodosii, Valentiniani, Martiani, Maioriani, Seueri libri V cum Aniani interpretationibus. Ex his etiam Nouellis multae tenebris auferuntur. Tituli ex corpore Codicis Gregoriani E Hermogeniani \& multo plures quam prioribus editionibus haberentur. Ex Gaii Institutionibus liber. Julii Pauli Receptarum Sententiarum ad filium lib. V cum Aniani interpretationibus \& auctiores \& castiores quam antea fuerint. Tituli ex corpore Vlpiani. Volusii Maetiani de partibus assis liber. Papiani liber responsorum qui etiam nondum fuerat editus. Item notae iuris a Magnone collectae. Haec omnia curante Iacobo Cuiacio, Lugduni, apud Guliel. Rovillium, 1566.

7 P. PiтHOU, Imperatorum Theodosii, Valentiniani, Maioriani, Anthemii nouellae constitutiones XLII nunc primum in lucem editae, Lutetiae, ex officina Roberti Stephani, 1571.

8 Codicis Theodosiani libri XVI. Imppp. Theodosii, Valentiniani, Martiani, Maioriani, Leonis, Seueri et Anthemii Nouellae Constitutiones. Tituli ex corpore Codicis Gregoriani. Tituli ex corpore Codicis Hermogeniani. Adiectae sunt suis locis Aniani V. S. interpretationes. XII Tabular. Fragmenta. Fragmenta ex libris Institutionum Gaii. Eiusdem Gaii Institutionum epitome. Volusii Metiani de asse liber singularis. Fragmentum ex Papiniani libro I Responsor. Iulii Pauli Sententiarum receptarum libri $V$ cum Aniani interpretationibus. Eiusdem Pauli Fragmentum ex li. II Institutionum. Domitii Vlpiani Regularum liber singularis. Fragmentum ex Modestini libro III Regularum. Lex Dei, siue Mosaicarum et Romanarum legum uetus collatio. Consultatio 
Parallelamente a quella del Codice Giustinianeo, fu dunque compiuta un'impresa di ricostituzione del Codice Teodosiano nella sua forma originale, che la tradizione manoscritta non consentiva di portare a completa perfezione, ma che, nell'arco di qualche decennio, grazie a delle ricerche largamente coronate da successo nelle biblioteche francesi e alla collaborazione di un piccolo gruppo di studiosi intorno al Cuiacio, riuscì a raggiungere un traguardo fondamentale. Il grande commento di Jacques Godefroy, pubblicato dopo la sua morte, si basava essenzialmente proprio sul lavoro editoriale di Cuiacio e dei suoi amici, e fu necessario attendere il diciannovesimo secolo e la scoperta di frammenti del Teodosiano in più manoscritti palinsesti e avvalersi delle ricerche sui manoscritti della lex Romana compiute da Gustav Haenel in tutte le biblioteche d'Europa perché la ricostituzione del Codice Teodosiano potesse fare nuovi passi innanzi, fino alla edizione di Mommsen, che tocca uno dei vertici della sua produzione scientifica. ${ }^{10}$

Resta che, da un punto di vista editoriale - ed è uno dei punti che bisogna tenere ben presenti - ci sono due collezioni distinte, quella del Teodosiano e quella della lex Romana Visigothorum, che non si sovrappongono se non per il fatto che la lex Romana Visigothorum resta la nostra fonte principale per i cinque primi libri del Teodosiano. L'edizione mommseniana del Teodosiano e delle Novelle ha largamente superato l'edizione che era stata preparata da Haenel per il Corpus edito da Boecking, ${ }^{11}$ ma per quel che concerne la lex Romana, l'edizione di riferimento resta un'altra edizione curata dallo stesso Gustav Haenel, nel 1849, che poté a giusto titolo proclamarsi Editio post

ueteris cuiusdam I. C. Fragmentum ex Dosithei Magistri grammatica. Stemma quemadmodum hereditates lege redeant. Burgundionis I. C. qui Papiani responsorum titulum praefert liber. Notae iuris a Magnone collectae. Notae Iuris ex Valerio Probo, Parisiis, apud Sebastianum Nivellium, 1586.

9 Codex Theodosianus cum perpetuis commentariis Jacobi Gothofredi... Opus posthumum... recognitum... opera et studio Antonii Marvilii..., Lugduni, sumptibus Joannis Antonii Huguetan \& Marci Antonii Ravaud, 1665.

10 Theodosiani libri XVI cum constitutionibus Sirmondianis et leges novellae ad Theodosianum pertinentes... Ediderunt Th. Mommsen et Paulus M. Meyer, Berolini, 1905.

${ }^{11}$ Codex Theodosianus. Ad LIV librorum manuscriptorum et priorum editionum fidem recognovit et annotatione critica instruxit Gustavus Haenel, Bonnae, apud A. Marcum, 1837-1842, in Corpus iuris Romani anteiustiniani consilio professorum Bonnensium Eduard Böcking, Moritz August von Bethmann-Hollweg et Eduard Puggé institutum, Bonnae, apud A. Marcum, 1837-1844. 
Sichardum prima. ${ }^{12}$ L'edizione di Du Tillet (1550), in effetti, si era limitata al Codice Teodosiano, rimpiazzando il testo della lex Romana con il testo completo dei libri 9-16 ed eliminando l'Interpretatio. Le due edizioni del Cuiacio raggruppavano l'insieme dei testi che costituivano la lex Romana Visigothorum, e continuavano a includere l'Interpretatio, ma vi aggiungevano altri testi - ci torneremo tra poco - e tentavano di ritrovare il testo originale del Teodosiano, così come delle Sententiae di Paolo. Erano, in certo modo, delle edizioni ibride, che facevano di tutto per ristabilire la situazione più antica e più completa dei testi giuridici utilizzati nella lex Romana, ma non rinunciavano ad includere un'Interpretatio che non aveva senso se non nel contesto della lex Romana. Questa scelta era coerente con la decisione del Cuiacio di includere nella raccolta del 1566 un liber Papiani responsorum, di cui Cuiacio non dubitava che fosse tardivo, e soprattutto di mantenere tale testo nella raccolta del 1586, benché nel frattempo Cuiacio e i fratelli Pithou si fossero resi conto che si trattava in realtà di una lex Romana Burgundionum, equivalente nella sua funzione per il regno dei Burgundi alla funzione per cui era stata emanata la lex Romana Visigotorum per il regno dei Visigoti. ${ }^{13}$ È vero che la pubblicazione delle leggi dei popoli germanici fu in gran parte opera dei medesimi giuristi (Sichard, Du Tillet, Pithou) che raccolsero il Ius anteiustinianum, ${ }^{14}$ ma ciò non toglie che le edizioni del 1566 e del 1586 abbiano segnato una svolta se paragonate al Codice Teodosiano edito da Du Tillet nel 1550 e alle Sententiae di Paolo edite dallo stesso Cuiacio nel 1558, in cui

12 Lex romana Visigothorum. Ad LXXVI librorum manu scriptorum fidem recognovit, septem ejus antiquis epitomis, quae praeter duas adbuc ineditae sunt, titulorum explanatione auxit, annotatione, appendicibus, prolegomenis instruxit Gustavus Haenel Lipsiensis. Editio post Sichardum prima, Lipsiae, Teubner, 1849.

13 Cfr. L. R. von Salis, Leges Burgundionum (Monumenta Germaniae Historica, Leges, I, II, 1), Hannoverae, impensis bibliopolii Hanhniani, 1892, p. 123-163 (Lex Romana siue Forma et expositio legum Romanarum).

14 J. Sichard, Leges Riboariorum Baioariorumque, quae uocant, a Theodorico rege Francorum latae. Item Alamannorum leges a Lothario rege latae, Basileae, 1530; [J. du Tillet], Libelli seu decreta a Clodoueo et Childeberto \& Clothario prius aedita, ac postremum a Carolo lucide emendata auctaque plurimum, poi successivamente Antiquae Burgundionum leges, Ripuariorum leges a Theodorico rege Francorum latae, Lex Alamannorum, Vetus lex Saxonum, Antiqua Baiuvariorum lex, s. l. n. d. (prima del 1557); P. Pithou, Edictum Theodorici regis Italiae, e Codicis legum Wisigothorum libri XII, Parisiis, apud Sebastianum Nivellium, 1579. 
aveva inserito un proprio commento in sostituzione dell'Interpretatio della lex Romana. ${ }^{15}$ E' interessante che i giuristi di Bonn guidati da Eduard Boecking, nel loro Corpus iuris romani anteiustiniani (nt. 10), abbiano anch'essi deciso di conservare l'Interpretatio, così come di mantenere l'Epitome Gai, nonostante che la scoperta del manoscritto di Verona di Gaio e la sua pubblicazione nel $1820^{16}$ avessero costituito, insieme all'apporto dell'epigrafia giuridica, l'evento determinante del rinnovamento nello studio del diritto romano. Fu Philipp Eduard Huschcke, nella sua Iurisprudentia anteiustiniana, ${ }^{17}$ che decise di escludere l'Interpretatio, e anche l'Epitome Gai, e Paul Krueger, Theodor Mommsen e Wilhelm Studemund fecero altrettanto nella loro Collectio librorum iuris anteiustiniani.$^{18}$ In seguito, peraltro, si è riconosciuto che l'Epitome Gai e l'Interpretatio fossero in larga misura una testimonianza del diritto romano insegnato nelle Scuole d'Occidente nel V secolo, ed esse hanno progressivamente e parzialmente rifatto la loro apparizione nelle raccolte di testi. ${ }^{19}$

Nel 1528, Sichard aveva deciso di completare il suo volume aggiungendovi testi che non si trovavano nella lex Romana ma che gli parevano rilevanti dal punto di vista giuridico (eo quo ad ius civile pertinerent): da una parte il piccolo trattato De asse di Volusio Meciano, un giurista dell'epoca degli Antonini, e d'altra parte due testi della raccolta dei Gromatici romani. Lidea di Sichard sarebbe stata ripresa e ampliata da Aimar de Ranconnet. Questo personaggio, Presidente al Parlamento di Parigi, era un grande erudito e vantava una collezione di manoscritti che, per le materie giuridiche, era considerata seconda soltanto a quella del re. La Bibliothèque Nationale de France conserva il suo esemplare del

$15 \mathrm{~J}$. CUJAS, Julii Pauli receptarum sententiarum ad filium libri $V$. In eosdem Iac. Cuiacii interpretationes, Parisiis, apud Andream Wechelum, 1558.

16 J. Fr. L. GösCHEN, Gaii institutionum commentarii IV... nunc primum editi, Berolini, G. Reimer, 1820.

17 PH. ED. HuschKe, Jurisprudentiae antejustinianae quae supersunt, Lipsiae, Teubner, 1861.

18 Collectio librorum iuris anteiustiniani in usum scholarum, ediderunt Paulus Krueger, Theodorus Mommsen, Guilelmus Studemund, Berolini, Weidmann, 1877-1890.

19 L'Epitome Gai nella nuova edizione della Jurisprudentia anteiustiniana curata da E. Seckel e B. Kübler (fascicolo II, 2, Leipzig, Teubner, 1927) ; l'Interpretatio delle Sententiae Pauli nei Textes de droit romain par Paul Frédéric Girard et Félix Senn, 7 édition par un groupe de romanistes, Paris, Dalloz, 1967. 
volume di Sichard, sul quale annotò una lista programmatica che, oltre ai testi editi da Sichard e ai Tituli Ulpiani, abbracciava l'intero corpus dei Gromatici e i trattati sui pesi e le misure di cui era a conoscenza, il trattato di Frontino De aquae ductibus, la Notitia dignitatum, infine i principali testi d'epigrafia giuridica allora conosciuti. ${ }^{20}$

Prima ancora di Sichard e di Ranconnet, fin dal 1513, il milanese Andrea Alciato, uno dei fondatori dell'umanesimo giuridico, aveva parlato della Notitia, e ne pubblicò una versione abbreviata nel 1529 e poi nel $1546 .{ }^{21} \mathrm{E}$ ' facile comprendere quale interesse, in un commentatore del Codice giustinianeo come Alciato, potesse suscitare questo quadro dell'alta amministrazione del tardo Impero romano. E lo stesso Alciato, nel 1518, aveva annunciato la sua intenzione di pubblicare vari testi gromatici. ${ }^{22}$ Ranconnet cercò di riunire dei manoscritti di tutti questi testi, e benché non abbia poi pubblicato nulla in prima persona, la sua biblioteca e i suggerimenti che elargì giocarono incontestabilmente un ruolo di primo piano nelle ricerche e nelle edizioni relative al Ius anteiustinianum. E' a Ranconnet che Jean du Tillet dedicò nel 1549 la propria edizione dei Tituli ex corpore Ulpiani, ${ }^{23} \mathrm{e}$ Cuiacio nel 1558 gli dedicò la sua edizione commentata delle Sententiae di Paolo (nt. 15). Nel 1564 di nuovo Cuiacio ricorderà che è in un manoscritto posseduto da Ranconnet che aveva trovato lo Stemma cognationum. ${ }^{24}$

Il grande progetto di Ranconnet non fu mai realizzato. La Notitia dignitatum fu pubblicata con il suo apparato iconografico nel 1552, da Sigismondo Gelenio, e nel 1554 Adrien Turnèbe e Pierre Galland diedero alle stampe con il loro apparato iconografico i prin-

20 BNF, Res F-381. Cfr. J.-L. FerRARY, Documents inédits relatifs à l'édition des fragments des XII Tables par Pierre Pithou (1586), in M. AsCHERI et G. COLLI (a cura di), Manoscritti, editoria e biblioteche dal medioevo all' età contemporanea. Studi offerti a Domenico Maffei per il suo ottantesimo compleanno, Roma, 2006, p. 311 e 327-328.

21 P.-Fr. GIRARD, Alciat et la Notitia dignitatum, in Studi in onore di S. Peruzzi nel XL anno del suo insegnamento, Palermo, 1925, p. 59-87.

22 A. Alciato, Paradoxorum ad Pratum libri VI, Dispunctionum libri IIII, In tres libros codicis libri tres, De eo quod interest liber unus, Praetermissorum libri II, Declamatio una, Mediolani, Alexander Minutianus, 1518 (Dispunctiones, III, 15).

${ }^{23}$ J. DU TIllet, XXVIIII Tituli ex corpore Vlpiani, Parisiis, apud Guil. Morelium, 1549.

${ }^{24}$ Iacobi Cuiacii I. C. Ad titulum Digestorum De excusationibus commentarius. Obseruationum lib. VI, VII, VIII, Lugduni, apud Guliel. Rovillium, 1564 (Obs. VI, 40). 
cipali testi di uno dei manoscritti gromatici. ${ }^{25}$ Questa edizione non era esaustiva, e soprattutto, era assai difettosa per quel che concerne i testi di carattere giuridico. Ne è la riprova l'esemplare che ne possedeva Pierre Pithou e che egli ha abbondantemente annotato, corretto, e completato. ${ }^{26}$ Cuiacio e i suoi discepoli seppero mettere a frutto il corpus gromatico. L'edizione del Teodosiano del 1566 incluse le quattro costituzioni del titolo De finium regundorum restituite appunto dal corpus gromatico e che erano state già pubblicate da Turnèbe e Galland senza rendersi conto - come rilevò Pithou - che questi testi del IV secolo non potevano per ragioni cronologiche essere attribuiti a un trattato di Frontino. Ma quest'aspetto del progetto di Ranconnet restò marginale per l'insieme dei lavori di Cuiacio e della sua cerchia.

Tornare a concentrarsi sui testi propriamente giuridici senza l'ampliamento suggerito da Ranconnet - era del resto suggerito dal moltiplicarsi stesso delle scoperte di testi giuridici. I Tituli ex corpore Ulpiani pubblicati dal Du Tillet nel 1549 (nt. 23), sebbene fossero stati trascritti dopo la lex Romana Visigothorum nell'unico manoscritto che ce ne è pervenuto, non facevano parte della lex Romana stessa. È il solo testo importante che Cuiacio abbia incluso nel 1566 nella sua prima edizione del Teodosiano, oltre a tre testi brevi: il De asse di Volusio Meciano già edito da Sichard, e le Notae iuris di Probo e di Magnone. Ma non incluse lo Stemma cognationum che aveva pubblicato due anni prima (nt. 24), e non pensò di approfittare dell'occasione per pubblicare la Consultatio veteris cuiusdam iuris consulti, di

25 S. GelEnIUS, Notitia utraque cum Orientis tum Occidentis ultra Arcadii Honoriique Caesarum tempora... Praecedit autem D. Andreae Alciati libellus De magistratib. civilibusque ac militaribus officiis... Cui succedit descriptio urbis Romae quae sub titulo Pub. Victoris circumfertur, et altera urbis Constantinopolitanae incerto auctore, nunquam antehac typis excusa... Subjungitur Notitiis vetustus liber de rebus bellicis ad Theodosium Aug. et filios ejus Arcadium atque Honorium, ut videtur, scriptus, incerto autore. Item... Disputatio Adriani Aug. et Epicteti philosophi, Basileae, apud Hier. Frobenium et Nic. Episcopium, 1552. - De agrorum conditionibus E constitutionibus limitum, Siculi Flacci lib. I, Iulii Frontini lib. I, Aggeni Vrbici lib. II, Hygeni gromatici lib. II. Variorum auctorum Ordines finitionum, De iugeribus metiundis, Finium regundorum, Lex Mamilia, Coloniarum pop. Romani descriptio, Terminorum inscriptiones E formae, De generibus lineamentorum, De mensuris \& ponderibus. Omnia figuris illustrata, Parisiis, apud A. Turnebum, 1554.

26 BNF, Res. F-891. 
cui aveva utilizzato il testo fin dal 1564 nel libro VII delle sue Observationes. ${ }^{27}$

Nel 1586 le cose sono molto cambiate. Due grandi testi indipendenti dalla lex Romana s'aggiungono ai Tituli Ulpiani: la Collatio, pubblicata nel 1574 da Pierre Pithou, ${ }^{28}$ e la Consultatio, che Cuiacio s'era infine deciso a pubblicare nel 1577. ${ }^{29}$ Alla Collatio, Pierre Pithou aveva aggiunto un brevissimo frammento di Modestino e i frammenti cosiddetti di Dositeo. Ma è da sottolineare soprattutto che Pierre Pithou aveva chiaramente concepito il progetto di riunire in un volume i frammenti dei giureconsulti e di destinare separatamente ad un altro le costituzioni imperiali. ${ }^{30} \mathrm{Il}$ progetto $\mathrm{fu}$ in definitiva realizzato dodici anni più tardi da Cuiacio e dai fratelli Pithou. Quest'edizione includeva ormai (con una sola eccezione) ${ }^{31}$ tutti i frammenti conosciuti di testi giuridici classici che non provenivano dal Corpus iuris civilis di Giustiniano. Soprattutto, conformemente al progetto concepito da Pithou nel 1574, il volume del 1586 è nitidamente diviso in due parti, ciascuna con una paginazione autonoma. In testa stanno le costituzioni

27 J. CuJAS, Obseruationum liber VII, cap. 5, 26 e 27 (nel volume citato nt. 24). Cfr. E. VOLTERRA, Il manoscritto della Consultatio ueteris cuiusdam iurisconsulti e il suo scopritore Antonio Loisel, in Scritti giuridici, IV, Napoli, 1993, p. 273-308.

28 P. PIтHOU, Mosaycarum et Romanarum legum collatio ex integris Papiniani, Pauli, Vlpiani, Gaii, Modestini, aliorumque ueterum Iuris auctorum libris ante tempora Iustiniani Imp. desumpta, Eiusdem Imp. Iustiniani Nouellae constitutiones III. Juliani antecessoris CP. dictatum de consiliariis. Ejusdem Juliani collectio de contutoribus. Ex bibliotheca P. Pithoei, Basileae, per Thomam Guarinum, 1574.

29 Opera Iacobi Cuiacii I. C. quae de iure fecit in hunc diem, ab ipso auctore disposita et recognita et aucta libro singulari Consultationum, et libr. XV, XVI, XVII Observationum, Paris, apud S. Nivellium, 1577, I, p. 271-280.

30 P. PITHOU, Mosaycarum et Romanarum legum collatio, p. 122: « hanc unam Modestini regulam ex alio codice qui patris V. C. fuit, pridem habuimus. Quae sequuntur » [cioè le Novelle di Giustiniano et i testi di Juliano l'Antecessore] « in eodem ipso exemplari erant in quo \& superiora omnia » [cioè la Collatio]. «Itaque quanquam nobis pridem in animo esset $\&$ haec alia quaedam ueterum Iurisconsultorum fragmenta uno uolumine complecti, altero Impp. constitutiones, quas etiam plures nondum editas nacti sumus, concludere, hic tamen adiicere uisum est interea dum res tempusque instituto nostro benignius adfuerint. Quod optare quidem quam sperare procliuius est ».

${ }^{31}$ I responsa di Adriano trasmessi da Dositeo e citati da Cuiacio nel 21 libro delle sue Observationes (in Jacobi Cujacii observationum lib. XXI, XXII, XXIII, XXIV. Ejusdem de origine juris ad Pomponium commentarius, et in libros IV Institutionum notae posteriores, Parisiis, apud S. Nivellium, 1585). 
imperiali (Codice Teodosiano; Novelle, ma anche i codici Gregoriano ed Ermogeniano, seguiti da copiosi indici e da una cronologia delle costituzioni). Nella seconda parte, intitolata « Veterum iuris consultorum reliquiae », si trovano i frammenti dei giuristi nell'ordine cronologico, che si tratti di veri frammenti oppure di testi più o meno profondamente rimaneggiati; poi vengono la Collatio, la Consultatio, i frammenti di Dositeo, lo Stemma, la lex Romana Burgundionum; su delle pagine non numerate, infine, si trovano dapprima i frammenti delle XII Tavole e infine le Notae di Magnone e di Probo. Questa divisione e quest'ordine si trovano anticipati in note manoscritte in due esemplari dell'edizione del 1566 che sono appartenuti a Pierre e a François Pithou, ${ }^{32}$ il cui ruolo dunque dev'essere stato decisivo per l'allestimento del volume del 1586. Come ho potuto dimostrare altrove, anche i frammenti delle XII Tavole, la cui presenza può sorprendere, sono un'iniziativa di Pierre Pithou. ${ }^{33}$

Nel corso del tempo, questa divisione fra costituzioni imperiali e scritti dei giuristi - che può sembrare un aspetto formale e secondario - porterà a una conseguenza notevole, cioè all'eliminazione delle costituzioni nelle raccolte che preferiranno chiamarsi Iurisprudentia anteiustiniana. Fu questa la scelta di Anton Schulting, che tuttavia conservò i due primi codici (il Gregoriano e l'Ermogeniano) ed escluse soltanto il Teodosiano e le sue Novelle; ${ }^{34}$ fu soprattutto la scelta di Huschke (nt. 17), che escluse tutti i Codici, ma fu peraltro costretto almeno a conservare le costituzioni che si trovano nella Collatio, nella Consultatio e pure nei Fragmenta Vaticana, scoperti nel 1822. Il Corpus di Bonn, sotto la direzione di Boecking (nt. 11), è l'ultimo a riunire tanto le costituzioni quanto gli scritti giurisprudenziali e pure se l'edizione dei singoli testi può sembrare superata, è questa completezza che determina il perdurante interesse di questa raccolta.

Possiamo trarre le fila di questo tragitto. Prima del Corpus iuris civilis ben delimitato di Giustiniano, non esisteva in effetti un Corpus iuris anteiustiniani altrettanto definito e unitario. Per tutto il periodo preclassico e classico, fino alla fine dei Severi nel III secolo d.C., la

32 Rispettivamente BNF, Res. F-3 e Res. F-2.

33 J.-L. FERRARY, Documents inédits (cit. nt. 20), p. 303-328

34 A. Schulting, Jurisprudentia vetus antejustinianea, Lugduni Batavorum, apud Jo. Vander Linden juniorem, 1717. 
nostra conoscenza del diritto romano riposa soprattutto sulla palingenesi dei giuristi romani, cioè sulla ricomposizione dei frammenti contenuti nel Digesto di Giustiniano, integrata con i frammenti contenuti nelle collezioni pregiustinianee così come con i rari brani trovati in manoscritti e papiri. Benché le Istituzioni di Gaio abbiano un carattere speciale e unico, essendo il solo testo classico che ci sia pervenuto nella sua integrità, è chiaro che quest'opera appartenga a questo stesso nucleo di testi giurisprudenziali. Un secondo insieme di testi del ius anteiustinianum corrisponde all'epoca intermedia fra il periodo della giurisprudenza classica e la compilazione di Giustiniano: in questo insieme la parte preponderante è rappresentata dai Codici di costituzioni, ma vi sono anche opere formate a loro volta da estratti di brani della giurisprudenza classica (le Sententiae di Paolo, i Tituli ex corpore Ulpiani, l'Epitome Gai) e altre raccolte che associano costituzioni e giuristi (come i Fragmenta Vaticana, la Collatio e la Consultatio). Il diciannovesimo secolo, che ha visto compiersi, grazie a Otto Lenel, il sogno di palingenesi degli scritti dei giuristi nato nel sedicesimo secolo a partire dall'opera di un altro discepolo di Cuiacio, l'Index legum di Jacques Labitte, ${ }^{35}$ assisterà anche alla disintegrazione del Corpus iuris anteiustiniani che Cuiacio e i fratelli Pithou avevano costituito pur senza dargli questo nome, e che s'era mantenuto fino all'impresa di Eduard Boecking.

Si tratta di una vicenda lunga e complessa, in cui si riflette il grande impegno intellettuale che vide generazioni di grandi studiosi francesi, italiani, tedeschi e di tutta Europa prestare le loro cure al patrimonio culturale costituito dal diritto di Roma.

35 O. Lenel, Palingenesia iuris civilis, Lipsiae, ex officina Bernhardi Tauchnitz,1889. J. LABITTE, Index legum omnium quae in Pandectis continentur, Parisiis, A. Wechelus, 1557. Cfr. J.-L. FERRARY, Le Digeste à l'envers. La palingénésie dans les travaux des juristes jusqu'à Lenel, in D. MANTOVAnI, A. PADOA SCHIOPPA (a cura di), Interpretare il Digesto. Storia e metodi, Pavia, IUSS Press, in corso di stampa. 